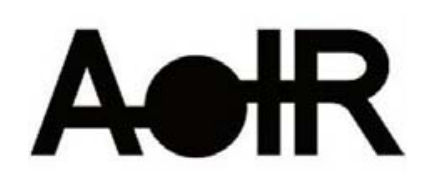

Selected Papers of \#AoIR2018: The $19^{\text {th }}$ Annual Conference of the Association of Internet Researchers Montréal, Canada / 10-13 October 2018

\title{
WHEN THE TEACHER BECOMES THE STUDENT: YOUTH IMPACT ON PARENT TECHNOLOGY USE
}

Jodi Dworkin, Ph.D.

University of Minnesota

Pooja Brar, M.S.

University of Minnesota

Heather Hessel, Ph.D.

University of Minnesota

We are only beginning to understand the ways in which young people are introducing technologies into the family system and how that is impacting family relationships. At this research stage, what seems clear is that face-to-face communication does not translate directly to the online context, and online communication is not completely replacing in-person communication. Youth and parents continue to report frequent inperson and phone communication (e.g., Connell \& Dworkin, 2012). Despite the lack of existing research considering the bi-directional relationships between technology use and family relationships, it is reasonable to expect that family relationships impact, in complex ways, how youth and parents use online media.

To advance the field, we need a deeper understanding of family relationships in the context of changing communication technologies. Researchers must begin to ask different questions using novel methodologies. The current study is designed to consider youth impact on parent technology use using family-level data.

\section{Socialization Theory}

Socialization theory describes how children internalize rules and norms from family, teachers, and media. Consistent with this perspective, most family studies on the uses and effects of media and communication have focused on how parents influence their children. Socialization however, is a bidirectional and reciprocal process in which not only are children socialized by their parents, but also actively socialize their parents Suggested Citation (APA): Dworkin, J., Brar, P., \& Hessel, H. (2018, October 10-13). When the teacher becomes the student: Youth impact on parent technology use. Paper presented at AolR 2018: The 19th Annual Conference of the Association of Internet Researchers. Montréal, Canada: AolR. Retrieved from http://spir.aoir.org. 
(Nelissen \& Van den Bulck, 2017). Despite there being many examples of this reciprocal process as part of the trajectory of normal adolescent development (for example, youth introducing music into the family system), researchers have lagged behind in the exploration of this dynamic. Children are active agents in the family, who can both intentionally and unintentionally influence parents (Crouter \& Booth, 2003; Kuczynski, 2003). Children socialize their parents into the use of media, which includes devices, as well as applications and tools such as texting.

\section{Method}

The current study used a sample of youth ( $37 \%$ female; $M$ age $=17.3$ years) and parents $(54.6 \%$ female; $M$ age $=41.5$ years) recruited from the same family. Parents were recruited online via MTurk, an online labor market; parent participants were asked to forward an invitation to participate to their youngest adolescent or young adult child. Participants were recruited from the U.S. and India; 98 parent-child dyads participated (34 from the U.S.; 64 from India).

\section{Measures}

In addition to reporting on demographic information, parents and children reported how often they used a variety of technology tools on a Likert-type scale from 1 (Never) to 6 (Several times a day). Six measures of technology use were considered in the current study: 1) general use to look for information, read news, and use online tools (general use), 2) audio or video calls (calls), 3 ) texting, instant messaging, discussion boards, or email (text), 4) sending or receiving audio or video files, and photos (audio and visual), 5 ) create or maintain blogs, microblogs, or websites (blogs), and 6) social networking sites (SNSs).

\section{Results}

First, a series of descriptive statistics, correlations, and t-tests were computed. For both Indian and U.S. families, parent and youth use of all tools were positively correlated $(p<.01)$.Child age (but not parent age) was positively correlated with child general use, child and parent calls, and child texting $(p<.05)$. T-tests revealed significant differences between U.S. and Indian families: child reports of general use $(t(1,91)=-2.76, p=.007)$, parent $(t(1,88)=-4.61, p=.000)$ and child $(t(1,90)=-2.18, p=.032)$ reports of calls, parent $(t(1,89)=-2.33, p=.022)$ and child $(t(1,86)=-2.41, p=.018)$ reports of texting, parent $(t(1,86)=-5.65, p=.000)$ and child $(t(1,89)=-3.21, p=.002)$ reports of blogs, and parent reports of audio and visual $(t(1,89)=-2.36, p=.020)$ were significantly higher among Indian families. Child report of SNSs was significantly higher among U.S. youth $(t(1,91)=2.21, p=.029)$.

Next, a series of six linear regression analyses were conducted with parent technology use as the dependent variable. Parent age, child age, parent gender, child gender, and country of origin were entered in the first step; child technology use was entered in the second step.

For general use $(\beta=0.197, t=2.334, p=.022)$, texting $(\beta=0.536, t=.853, p=.000)$, and SNS $(\beta=0.450, t=3.468, p=.001)$, only child use was significant in the full model, accounting for $6.0 \%, 27.0 \%$, and $12.5 \%$ of the variance in parent use, respectively. 
In the analyses for audio and visual, country of origin $(\beta=2.127, t=2.404, p=.019)$ and child use $(\beta=0.504, t=4.616, p=.000$, child use accounted for $18.4 \%$ of the variance in parent use) were significant in the full model. Being from the U.S. was associated with greater parent use. Similarly, in the analyses for blogs, country of origin $(\beta=0.504$, $t=4.616, p=.000)$ and child use $(\beta=0.528, t=5.977, p=.000$, child use accounted for $21.4 \%$ of the variance in parent use) were significant in the full model. Being from the U.S. was associated with greater parent use.

In the analyses for calls, child age $(\beta=0.225, t=2.228, p=.028)$, parent gender $(\beta=-1.363$, $t=-2.249, p=.027)$, country of origin $(\beta=2.003, t=3.171, p=.002)$, and child use $(\beta=0.336$, $t=3.370, p=.001$ ) were significant in the full model. Child use accounted for $8.4 \%$ of the variance in parent use. Being female, from the U.S., and for children being older was associated with greater parent use.

\section{Discussion}

Despite the small sample size in the current study, it is quite clear that child technology use is strongly associated with parent use, even when considering the diversity of ways individuals use technology - child use accounted for more than $20 \%$ of the variance in parent use of technology to create or maintain blogs, microblogs, or websites, and use of technology for texting, instant messaging, discussion boards, or email.

New technologies that are introduced to the family system are a source of social change, whose effects are mediated by the ways in which the media are processed by individual family members. Family stability will be determined by how the family system responds to these changes that are brought into the system by youth (Rudi et al., 2015). Socialization theory is one lens that moves the field towards better understanding the bidirectional relationships between parents and children. Future research should use longitudinal data to explore how children impact parents' technology use over time how that influence changes with age, sociohistorical time and place, and life transitions. Longitudinal data would also allow for deeper exploration of the conditions under which children and parents mutually influence each other's technology use and the influence of this complex dynamic on family relationships. Finally, future research should consider how children's use of technology impacts parent technology use across cultures.

\section{References}

Connell, J., \& Dworkin, J. (2012). College students' information and communications technology (ICT) use with parents. Journal of the Association of Higher Education Parent/Family Program Professionals, 2(2), 2-17.

Crouter, A. C., \& Booth, A. (2003). Children's influence on family dynamics: The neglected side of family relationships. Lawrence Erlbaum: Mahwah, NJ.

Kuczynski, L. (2003). Beyond bidirectionality: Bilateral conceptual frameworks for understanding dynamics in parent-child relations. In L. Kuczynski (Ed.), Handbook of dynamics in parent-child relations (pp. 1-24). Thousand Oaks, CA: Sage. 
Nelissen, S., \& Van den Bulck, J. (2017). When digital natives instruct digital immigrants: active guidance of parental media use by children and conflict in the family. Information, Communication \& Society, 38, 1-13.

Rudi, J. H., Dworkin, J., Walker, S. K., \& Doty, J. L. (2015). Parents' use of information and communications technologies for family communication: differences by age of children. Information, Communication and Society, 8(1), 78-93. 